\title{
Performance Enhancements for Asynchronous Random Access Protocols over Satellite
}

\author{
Christian Kissling \\ German Aerospace Centre (DLR) \\ Oberpfaffenhofen, D-82234,Wessling, Germany \\ Email: christian.kissling@dlr.de
}

\begin{abstract}
In this paper, a novel enhancement of the well known ALOHA random access mechanism is presented which largely extends the achievable throughput compared to traditional ALOHA and provides significantly lower packet loss rates. The novel mechanism, called Contention Resolution - ALOHA (CRA), is based on transmitting multiple replicas of a packet in an unslotted ALOHA system and applying interference cancellation techniques. In this paper the methodology for this new random access technique is presented, also w.r.t. existing Interference Cancellation (IC) techniques. Moreover numerical results for performance comparison with state of the art random access mechanisms, such as Contention Resolution Diversity Slotted ALOHA (CRDSA) are provided. Finally the benefit of taking strong forward error correcting codes for the performance of CRA is shown.
\end{abstract}

\section{INTRODUCTION}

The usage of random access mechanisms for satellite communication has received increased attention again in the recent past. While techniques based on Demand Assigned Multiple Access (DAMA) can on one side allow an efficient usage of the available system resources for high-duty cycle and predictable traffic, DAMA techniques also show several drawbacks, especially for unpredictable and bursty traffic, such as in the internet or in Air Traffic Management (ATM). A general advantage of DAMA based systems is that the resource manager always has full overview and control of the system resources and can decide how these are used. In particular, the operating point of the DAMA channel can be controlled and congestion situations can be dealt with in a controlled manner. DAMA protocols show good performance in cases where medium to large data volumes have to be transmitted or for traffic sources which show a periodic (predictable) behaviour or a high duty cycle where the advantage of Predictive Capacity Estimation (PCE) becomes even more apparent. On the other hand, DAMA protocols have several drawbacks such as additional delay due to the signalling procedure and the overhead of the signalling messages. For application in e.g. a Geostationary Orbit (GEO) satellite scenario, the latency introduced by DAMA capacity request signalling sums up to 1 Round Trip Time (RTT), which equals to $\approx 500 \mathrm{~ms}$, before the transmission can actually start. Such an additional delay may not be too critical for a wide range of applications as existing in the terrestrial internet (e.g. HTTP, email) but for other applications, such as safety critical aeronautical communication (for instance exchange of information between pilots and controllers on ground), this additional delay can get critical for meeting the operational requirements, e.g. the maximum message expiration time (see [1] for further details). Random Access (RA) schemes are therefore being considered an interesting candidate for this low-duty cycle traffic [11], avoiding the signalling overhead of DAMA and providing short transmission latencies, since avoiding the signalling RTT. RA schemes have a long history and were a topic for research in all different facets. Starting from the initial publication of ALOHA by Abramson [2] a vast amount of evolutions of RA protocols can be found in the literature. In the following only the ones which are most relevant for this work shall be reviewed for pointing out the novelties and difference of the new CRA scheme proposed in this work with respect to them. The original proposal of ALOHA was done for sharing a broadcast channel by multiple users in an asynchronous manner, i.e. without the need to have the users synchronized to any time reference or organizing the channel access among them. In ALOHA, users transmit their packets at random times and any collisions among two or more transmissions results in a loss of the collided packets. As is well known, the ALOHA scheme reaches a maximum achievable throughput of $T=e^{-2} \frac{p k t}{\text { slot }} \approx 0.18 \frac{p k t}{\text { slot }}$ due to this. The second well known evolution followed by Roberts in [3]. In this work the main innovation w.r.t. ALOHA was the segmentation of time into slots and restrictions that packet transmissions may only happen at the beginning of a slot and that a packet transmission lasts exactly one slot. This way the throughput of the so called Slotted ALOHA (SA) protocol increased to $T=e^{-1} \frac{p k t}{\text { slot }} \approx 0.36 \frac{p k t}{\text { slot }}$. Another method which was also proposed in [3] was to exploit the capture effect in situations where a collision occurs in a slot but where the receiver is able to capture the signal of the stronger user (due to sufficient power unbalance) to correctly receive at least one of the conflicting packets. After many publications investigating the performance of the SA protocol in different conditions, stability behaviour and retransmission strategies [4]-[7], a further generalization of SA, named Diversity Slotted ALOHA (DSA) [8] followed. Here, instead of transmitting a packet in a single burst, a user transmits several copies of the same packet in different bursts. It was found there that the performance of the transmission delays can be improved for light traffic load conditions w.r.t. SA and that if a maximum number of retransmissions for lost packets is given, then multiple 
transmissions result in a higher throughput. Besides the RA evolutions of combining SA with spread spectrum techniques, recent work has focused on evolutions of Successive Interference Cancellation (SIC) applied to the SA protocol [9][12]. The fundamental concept of this SA enhancement is to try to recover additional packets by successive cancellation of interference. To do this (in simplified words) the slots are grouped into frames of $N_{S}$ slots. Instead of a single packet transmission as in SA, every terminal transmits additionally one (CRDSA) or more (CRDSA++) replicas of the packet in different slots. Hereby every burst header contains a pointer to the replica location(s). If a burst can be decoded, the channel is estimated from it (e.g. with the method described in [9]), the location of the replica(s) is extracted from the header and the interference caused by the packet is removed from the other slot(s) under consideration of the channel estimation. This way the interference caused to burst(s) transmitted in other slot(s) is reduced so additional bursts can possibly be also decoded in the subsequent iterations. Performance evaluations within [9][12] have shown that the maximum throughput (normalized to slots) can be impressively extended from $T_{S A} \simeq 0.36 \frac{\mathrm{pkt}}{\text { slot }}$ up to $T_{C R D S A} \simeq 0.55 \frac{p k t}{s l o t}$ when transmitting one replica (degree=2) and even up to $T_{C R D S A++} \simeq 0.68 \frac{p k t}{s l o t}$ for degree 4 (3 replicas). Additionally, the Packet Loss Rates (PLR) drop down to very low values. While SA meets a PLR of $10^{-3}$ for a maximum offered (normalized) traffic load $G_{\max }=10^{-3} \mathrm{Erl}$, the CRDSA scheme can meet the same PLRs for a maximum offered traffic load of $G_{\max }=5.5 \cdot 10^{-2} \mathrm{Erl}$ and CRDSA-4 ${ }^{1}$ even for $G_{\max }=0.6 \mathrm{Erl}$. A positive effect of these low PLRs is that much fewer retransmission are necessary to meet an overall end-to-end PLR. Furthermore the additional gain by exploiting power unbalance between users is investigated in [10] - [12], showing that the system performance can benefit from power unbalance. Most recently, the CRDSA protocol was further extended and optimized by applying variable burst repetition rates which are based on bipartite graph optimization [13], [14]. Within [13] and [14], the iterative interference cancellation process is described by means of a bipartite graph, similar to the erasure decoding of graph-based codes. It is also shown there how the throughput and PLR performance can be increased by selecting the repetition degree from a probability distribution, resulting in variable repetition rates. By optimization of the probability distributions, e.g. by means of differential evolution, the maximum throughput of the so called Irregular Repetition slotted ALOHA (IRSA) can be increased up to $T_{I R S A} \simeq 0.97$.

While CRDSA and IRSA represent a SIC framework for SA, the concept of SIC was not yet applied towards plain ALOHA. This work thus focuses on defining a framework for the application of SIC techniques to plain ALOHA (see section II) and provides a simulative performance evaluation of the new CRA protocol (section III) and the Irregular

\footnotetext{
${ }^{1}$ For nomenclature, the total degree of packet transmissions is indicated behind the protocol, so CRDSA-4 refers to transmitting 1 burst +3 replicas, i.e. a total of 4 bursts
}

Repetition Contention Resolution ALOHA (IRCRA) extension of it, using variable repetition rates, in different conditions. As will be shown in the remainder of this work, the proposed CRA protocol offers several advantages compared to CRDSA and IRSA and boosts the performance significantly compared to plain ALOHA. The simulation results presented in section III also confirm the assumption that CRA can benefit from strong Forward Error Correction (FEC) coding also in perfect power balance conditions, whereas CRDSA and IRSA can only benefit significantly in presence of power unbalance among transmissions.

\section{SyStem OVERVIEW AND PROPOSED ENHANCEMENTS}

The scenario under consideration here consists of several user terminals communicating over a satellite to a ground station (gateway). In contrast to the slotted protocols, where terminals need to be synchronized on per slot basis, the terminals here only synchronize to the time frames. In contrast to plain ALOHA, a packet is not sent directly upon generation by the higher layers, but at a random time (uniformly selected) within the frame. For every packet arrival, a random transmission time within the frame is selected. In addition, every packet is not only sent once, but is replicated $d-1$ times. The total number of times a packet is sent in a frame is called the degree (hereafter referred to as $d$ ). In order to avoid self-interference the transmission times of the replicas are chosen by each transmitter so they do not overlap. Within the scope of this work, all packets are considered to have the same duration $\tau$. This procedure is followed by all terminals, resulting in the situation shown exemplary in Figure 1(b). In order to highlight the differences of CRA w.r.t. CRDSA, the methodology of CRDSA is quickly reviewed. Figure 1(a) shows the IC process for CRDSA. The bursts which are erased are illustrated with a grid pattern. As can be seen only the first burst of user 1 arrives with no collisions. In CRDSA the first burst of user 1 is decoded and with the knowledge of the content of this burst, the interference of user 1 on user 2 in slot 5 is removed. In the second iteration, now packet 2 can be decoded and its interference on the packet of user 3 (slot 2) can be removed. After all iteration rounds, all packets can be decoded in this example. For Contention Resolution - ALOHA (CR-ALOHA) the scenario is illustrated in figure 1(b). Since no discrete time slots are used here anymore, the burst arrivals happen at random times within the frame, whereas the arrival time is uniformly distributed in the range $t_{\text {arr }} \in\left[t_{0} ; t_{0}+T_{F}-\tau\right]$ whereas $t_{0}$ denotes the beginning of the frame and $T_{F}$ the duration of the frame. As consequence partial interference and erasure can occur. In the remainder of this work, two cases are distinguished for this reason. In the first case, any overlap is assumed to result in erasure and loss of the total packet. In the second case, the application of strong FEC is considered which allows decoding of the packet even in presence of (limited) interference. In the first case, only packet 1 can be decoded, and its interference on user 2 is removed. In the following iteration round, packet 2 can be decoded now and its interference on user 3 can be removed. 
Finally also in this example all packets can be received. The process of IC is repeated until either all packets have been recovered or until a maximum number of iterations $I_{\max }$ has been reached. For the second case, the possibility to decode a burst depends on the actual amount of interference power. Apart from this the procedure is the same. In the simulations presented afterwards, packet recovery is done jointly at the end of every frame and not on the fly while processing the frame, so decoding iterations refer to full frame processing rounds.

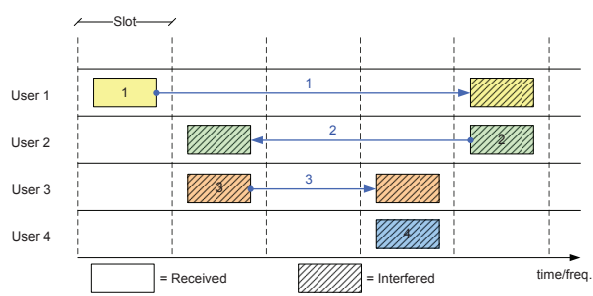

a) CRDSA IC $(\operatorname{deg}=2)$

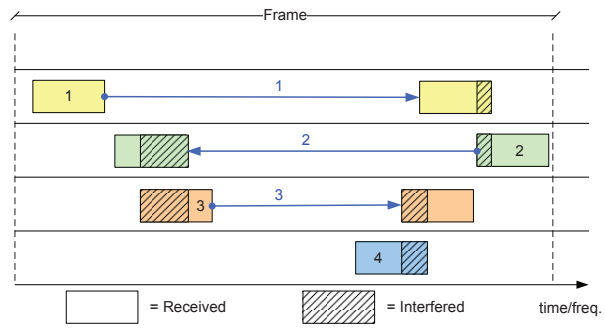

b) New CRA IC $(\mathrm{deg}=2)$

Fig. 1. CRDSA (a) and CRA (b) interference at beginning of IC process

Finally, the concept of CRA was further extended in the light of the evolution of CRDSA towards IRSA. Instead of fixing a burst degree $d$, the actual number of replicas is selected from a probability distribution which is represented in a polynomial way by $\Lambda(x)$ [13],[14]. In this case every terminal selects the repetition rate $d$ with probability $p_{d}$ from $\Lambda$.

$$
\Lambda(x)=p_{1} \cdot x+p_{2} \cdot x^{2}+p_{3} \cdot x^{4}+\ldots+p_{d_{\max }} \cdot x^{d_{\max }}
$$

As was shown in [14], the use of such a Probability Density Function (PDF) can further increase the performance of the IC scheme. Within this work, the optimization of the PDF for IRCRA is out of scope so the PDF provided in [14] is used for the CRA simulations presented later.

\section{A. Advantages of proposed method}

The proposed extension of ALOHA for contention resolution has, from a system perspective, several advantages, which shall be elaborated a bit more in detail in the following.

a) Relaxation of timing requirements: In contrast to slotted schemes such as SA, CRDSA (and extensions of CRDSA) and IRSA the proposed approach does not require precise synchronization of users on a time slot basis, but only on long time frame basis. This means a significant relaxation of synchronization and timing requirements w.r.t. SA and CRDSA. b) Avoiding size limitations: The restriction of a defined packet size does not exist in the proposed CRA. In the slotted schemes, the maximum burst size is given by the length of a slot and its peak burst rate. This hard limitation can result in the need for fragmentation of the data packets and consequently in the need of re-ordering fragments prior to defragmentation because fragments will not be delivered ordered due to the SIC process. In CRA on the other hand packet lengths can vary. Fragmentation can thus be avoided in many cases.

c) Benefitting from FEC even in absence of power unbalance: While in the slotted schemes the interference is always entirely interfering a burst or not interfering at all, in the proposed scheme the interference can be (and in most cases will be) only partial. The application of FEC coding can thus boost the performance of CRA in situations where the performance of the slotted schemes does not benefit from FEC such as the case of no or limited power unbalance among different users.

\section{B. Simulation approach}

In order to evaluate the performance of the newly proposed CRA algorithm and show the gains in throughput and lower PLR, numerical simulations have been performed. For the simulations, ideal channel estimation and IC have been assumed in the following. In the considered scenario, the system is populated by $N_{u}$ users, generating traffic. Every packet generation results in $d$ transmitted bursts, so $d$ is the replication factor. For the case, where FEC is considered to counteract partial interference, a rate $R$ is applied, with $R=R_{c} \cdot \log _{2} M$ being $R_{c}$ the coding rate and $M$ the modulation index.

The normalized offered traffic load is given by $G=\frac{N_{u} \cdot \tau}{T_{F}}$ and the normalized throughput $T$ is defined as the probability of successful reception of a packet, multiplied by the offered traffic load $G$, in formulas $T(G)=G \cdot \Psi_{\Omega}(G)$. The normalized throughput here is related to the logical throughput, i.e. user packets, whereas the physical throughput would also consider the number of replicas generated per packet. The success probability $\Psi_{\Omega}(G)$ is hereby a function of the offered load $G$ and the set of system parameters $\Omega$ which consists of $\Omega=\left\{T_{F}, d, R, I_{\max }, S N I R\right\}$

For the analysis carried out in this paper, the decoding threshold is approximated with the Shannon bound given by $C=\log _{2}(1+S N I R)$. When setting $C=R=$ $\log _{2}(1+S N I R)$ this results in the required decoding threshold $S N I R_{d e c, d B}=10 \cdot \log _{10}\left(2^{R}-1\right)$. In case the resulting SNIR for a packet is falls below the decoding threshold $S N I R_{d e c, d B}$ the packet replica is considered lost, while if the SNIR is exceeding the threshold it is considered as correctly decoded. This is clearly a simplified approach which is however valid for moderate to high SNIR if properly designed FEC schemes are used. Future work, already started at the moment is dealing with the use of the performance curves of real codes.

A frame length of $T_{F}=100 \mathrm{~ms}$ and packet lengths of $\tau=1 \mathrm{~ms}$ were selected. This has been done to have comparable results with the simulations for CRDSA-x, in 
which the same frame length and time slots with $1 \mathrm{~ms}$ duration are used. The maximum decoding iteration for CRA was set to $I_{\max }=20$. The behaviour was investigated for different repetition rates $d=2,3,4$ and using QPSK as modulation scheme with a FEC 1/2. As explained before, the theoretical Shannon bound was used as decoding threshold. Future work will deal with the implementation of real existing codes. For the SNR, two scenarios with $S N R_{1}=10 d B$ and $S N R_{2}=2 d B$ were simulated. Last not least, no power unbalance has been considered in this work. Future work is dealing with investigation of the CRA performance also in presence of power unbalance.

\section{NUMERICAL RESULTS}

\section{A. No FEC}

The first set of simulations considers the system without FEC (case 1). For CRA, this means that any interference (i.e. any overlapping in time among two transmission trials) results in a loss of the entire packet. In case of slotted schemes (SA, CRDSA, IRSA) the two possible interference cases are a full collision, resulting in loss of all collided packets in this slot and no collision, resulting in successful reception of the uninterfered packet. Figure 2 shows the simulated throughput of the system, comparing a plain ALOHA, a plain SA and CRDSA with repetition degree $d=2$, with the proposed CRA with repetition degrees $d=2,3,4$ and with IRCRA, $\Lambda_{0}(x)=0.5 \cdot x^{2}+0.28 \cdot x^{3}+0.22 \cdot x^{8}$. It can be observed

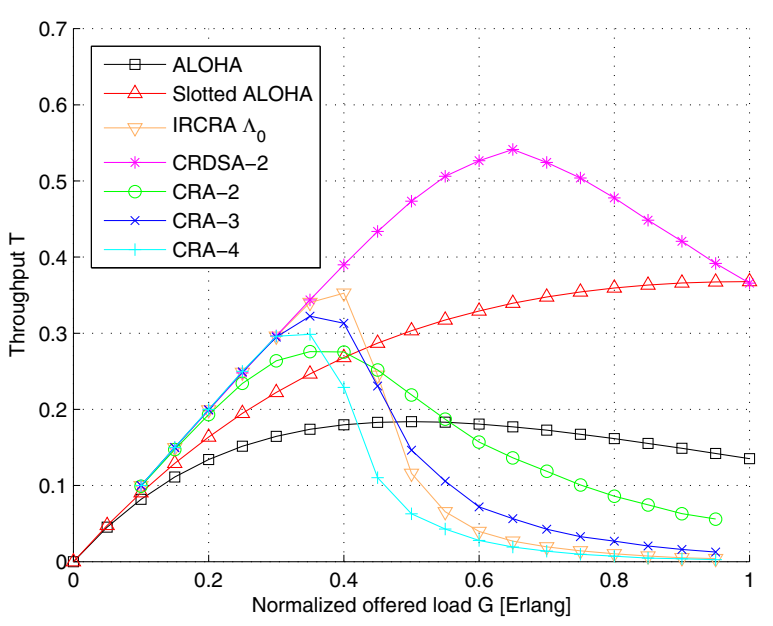

Fig. 2. Throughput performance without FEC

from figure 2 that the throughput of CRA w.r.t. the normal ALOHA is almost doubled, from a maximum of $18 \%$ for ALOHA to $32 \%$ for CRA-3. Furthermore the throughput is almost linear in the region up to $G=0.3 \mathrm{Erl}$, compared to the nonlinear behaviour of ALOHA without IC. Looking at the PLR in figure 3, an interesting observation is that the PLR of CRA-3 and 4 even falls below the one of CRDSA-2 for low offered traffic load $G<0.3$. Finally it can be observed that the performance (throughput and reduction of PLR) can be further boosted by applying the irregular repetition rates for IRCRA. This makes the CRA protocol especially interesting

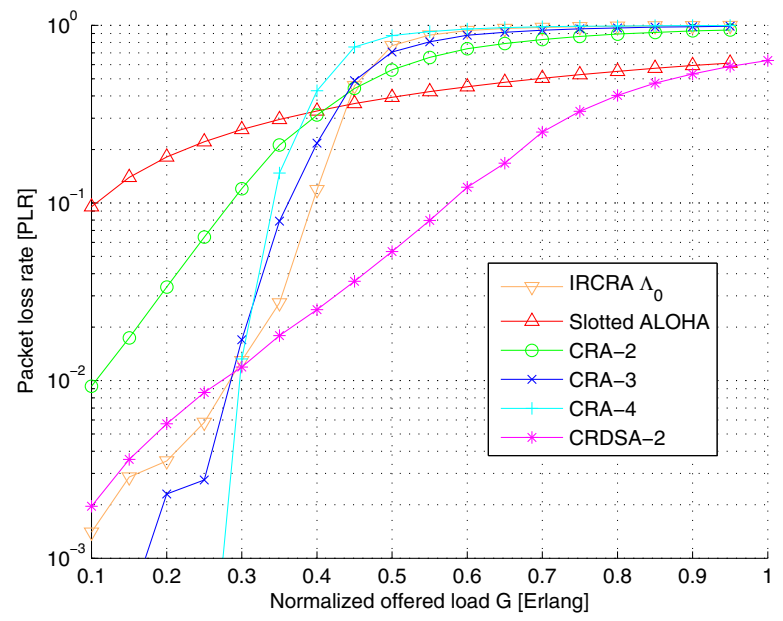

Fig. 3. Packet loss rates without FEC

for application in scenarios where very low traffic load is generated and where the terminal equipment cannot achieve the precise slot synchronization required by CRDSA or IRSA.

\section{B. $S N R=10 d B, F E C 1 / 2$}

In this and the next section, the performance of the new CRA protocol is investigated in the presence of coding (case 2). In [10], the performance of CRDSA was investigated in the presence of FEC and power unbalance among users. As a baseline, a $S N R=10 d B$ together with QPSK and FEC $1 / 2$ was chosen for the comparison there. On the basis of these values, also the first set of performance computations for CRA is done here. Figure 4 shows the simulation results for the normalized throughput in this scenario. The most

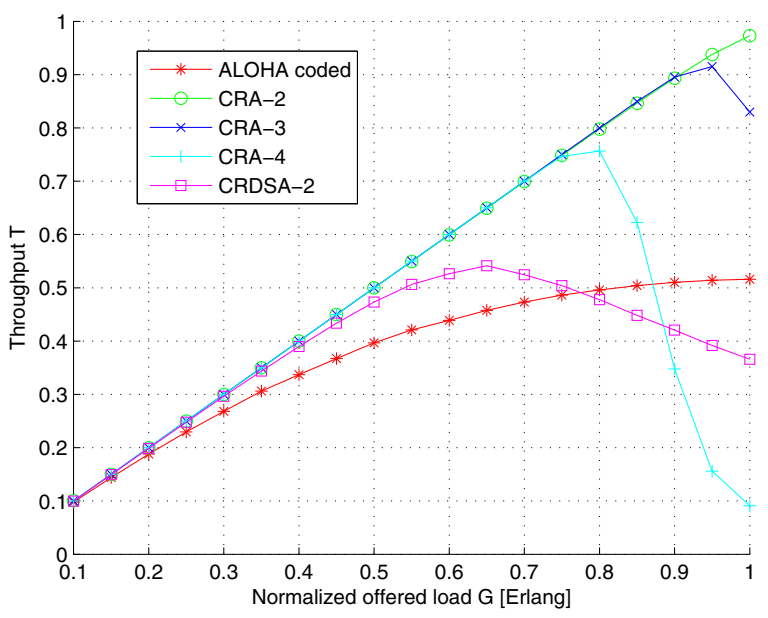

Fig. 4. Throughput vs. normalized load for $S N R=10 d B$, QPSK, FEC $1 / 2$ Shannon decoding thresholds

interesting observation is that the newly proposed CRA largely 
outperforms even the CRDSA-2 protocol, reaching extremely high throughput values, almost linear along the full range of offered loads. For higher repetition degrees, the throughput performance of CRA reaches then a maximum of $T=0.92$ for repetition degree 3 and $T=0.75$ for repetition degree 4. While it should be noted that these results are for a scenario with a high Signal-to-Noise ratio (SNR) dimensioning and strong coding, it nevertheless represents an outstanding improvement compared to existing random access protocols in same conditions. A look at the resulting PLR as shown in figure 5 also shows that the PLR, in particular for CRA-2 stays very low, even at very high normalized offered traffic loads.

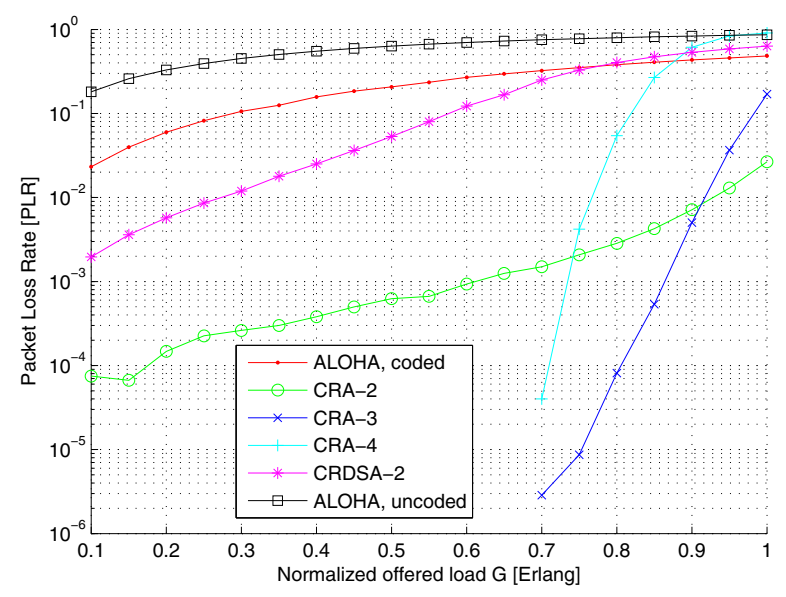

Fig. 5. Packet Loss Rate vs. normalized load for $S N R=10 d B$, QPSK, FEC 1/2 Shannon decoding bound

One reason for this excellent result can be found in the following considerations. Assuming the Shannon limit as decoding bound, the required minimum SNIR computes to

$$
S N I R=\frac{P}{x \cdot P+N}
$$

whereas $x$ is the interference ratio ( $x=0$ corresponds to no interference, $x=1$ to full interference with one other user, $x=2$ to full interference with two other users, etc.). The SNIR in dependence of the interference ratio is also shown in figure 6. The Shannon decoding bound assuming QPSK modulation and code rate $1 / 2$ computes to $S N I R=2^{R}-1=1$ to $0 \mathrm{~dB}$. For slotted schemes, the only two possible interference cases are no collision in a slot and collision with at least one other user. In the example above this results in an SNIR of $10 d B$ (no collision) and $S N I R \leq-0.41 \mathrm{~dB}$ for collision with at least one further user. Comparing this to the decoding bound means that the slotted schemes cannot benefit from a high SNR, except in cases of power unbalance between users. The unslotted CRA on the other hand can tolerate interference up to $90 \%$ before crossing the decoding bound as can be also seen in figure 6.

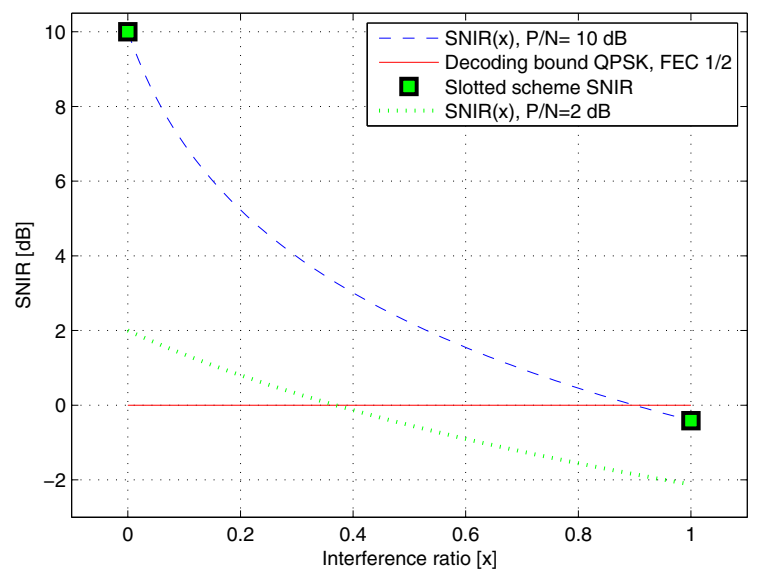

Fig. 6. SNIR as function of interference ratio for unslotted (blue) and slotted (green) schemes for $P / N=10 d B P / N=2 d B$ and QPSK, FEC $1 / 2$

\section{C. $S N R=2 d B, F E C 1 / 2$}

To investigate the performance of the CRA protocol in a more challenging environment, the SNR was decreased to $2 d B$. Figure 7 shows the results for the normalized throughput in this scenario. As can be seen, the maximum through-

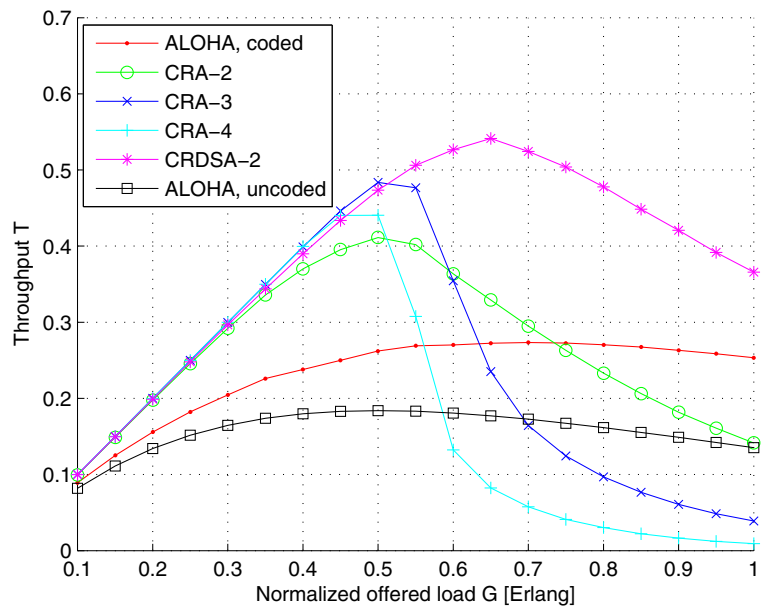

Fig. 7. Normalized throughput vs. normalized load for $S N R=2 d B$, QPSK, FEC 1/2 Shannon decoding bound

put of CRA is largely reduced compared to the previous scenario with $S N R=10 \mathrm{~dB}$, but still comparatively high with $T_{\max , 3}=0.48$ for CRA-3. The high linearity up to $G=0.5 \mathrm{Erl}$ for degrees 3 and 4 and up to $G=0.3 \mathrm{Erl}$ is also remarkable. The resulting PLRs in figure 8 , also show still a very good behaviour, going down to PLR $10^{-4}-10^{-5}$ for CRA-3 and 4 and for low values of the normalized offered load $G$. One reason for the performance drop compared to the previous scenario is the change of tolerable interference ratio, as shown in figure 6. While for $S N R=10 \mathrm{~dB}$ interference ratios up to $90 \%$ can be tolerated, this ratio drops to $36 \%$ 


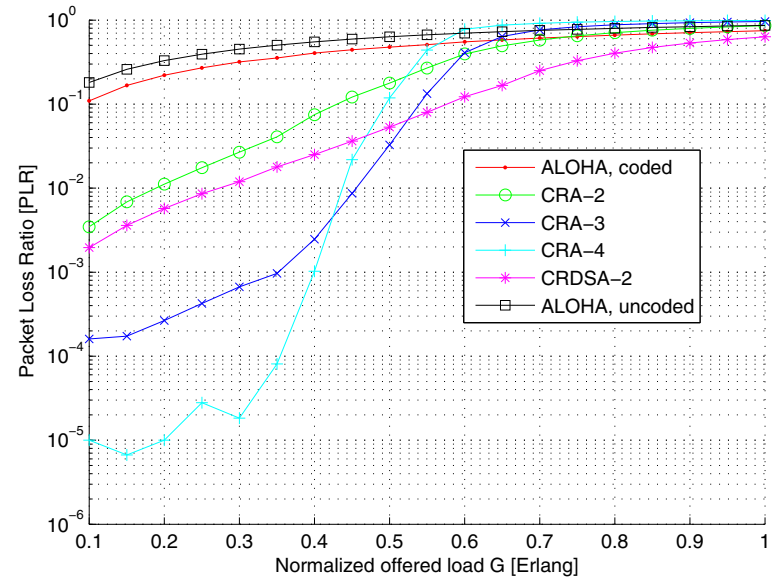

Fig. 8. Packet loss rate vs. normalized load for $S N R=2 d B$, QPSK, FEC $1 / 2$ Shannon decoding bound

for $S N R=2 d B$, which explains the reduced PLR and throughput performance.

\section{CONClusions AND Future WORK}

In this paper a new random access protocol for unslotted medium access was introduced. Conceptually an unslotted RA protocol offers several advantages over a slotted one. While in a slotted scheme exact timing on a burst basis is required, a synchronization on frame level is sufficient for the proposed CRA protocol. In quantitative numbers this means a relaxation of the timing demands easily reaching the order of a factor of $100\left(T_{F}=0.1 \mathrm{~s}\right.$ compared to $\left.T_{s}=10^{-3} \mathrm{~s}\right)$. Additionally, slotted protocols have a hard limitation for the information that can be carried in a burst. For varying packet lengths this can result in the need of fragmentation, creating in turn the need to deal with in-order delivery and a policy definition for fragment-to-frame assignment. The presented unslotted CRA protocol on the other hand also supports packets with varying packet lengths, avoiding fragmentation in many cases. Finally the unslotted schemes can benefit better from high SNRs also in the case of power-balance, since partial collisions result in variable interference ratios, while in the slotted schemes there is either no collision (i.e. no interference) or a collision (meaning full interference) in a slot. Besides these general advantages, the numerical results have shown that the CRA protocol is able to operate at very low PLRs below $10^{-2}$ going even down to $10^{-5}$ in presence of FEC. Even in absence of coding, the proposed protocol can outperform the slotted RA protocols in terms of PLR for low offered traffic loads, while still offering the advantages mentioned above. Also with respect to the offered throughput, the CRA protocol shows a significant performance enhancement, boosting the performance of plain ALOHA $\left(T_{\max } \approx 18 \%\right)$ to $T_{\max , C R A} \approx 36 \%$ even in absence of coding. In presence of coding and dependent on the SNR, the packet throughput performance is linear up to very large values of offered load $G$. All the mentioned advantages show that the CRA protocol is a highly interesting candidate which can ease the system design requirements while keeping or even increasing the performance compared to a slotted RA scheme. Considering the possibility to benefit from partial interference, the CRA scheme may also prove interesting in scenarios of power unbalance among users. Work going on at the moment is investigating this aspect, as well as the possible performance enhancements of using optimized irregular packet repetition rates. Additionally, the usage of real codes instead of the theoretical Shannon bound and the effect of power unbalance are under investigation.

February 7th, 2011

\section{ACKNOWLEDGMENT}

The author would like to thank Gianluigi Liva for providing his expertise and comments in many fruitful and productive discussions.

\section{REFERENCES}

[1] Eurocontrol, FAA, "Communications Operating Concept and Requirements for the Future Radio System (COCR), version 2.0.

[2] N. Abramson, "The ALOHA system: Another alternative for computer communications", Proceedings of the 1970 Fall Joint Comput. Conf., AFIPS Conf., vol. 37, pp. 281-285, Montvale, N. J., 1970.

[3] L. G. Roberts, "ALOHA packet system with and without slots and capture", Comput. Commun,. Review, vol. 5, pp. 28-42, April, 1975.

[4] A. B. Carleial and M. E. Hellman, "Bistable behaviour of ALOHA-type systems", IEEE Transactions on Communications, vol. COM-23,pp. 401410, April, 1975.

[5] L. Kleinrock, S. S. Lam, "Packet switching in a multiaccess broadcast channel: Performance evaluation", IEEE Transactions on Communications, vol. COM-23, pp.410-423, April, 1975.

[6] M. J. Ferguson,'On the control, stability and waiting time in a slotted ALOHA random-access system", IEEE Transactions on Communication, vol. COM-23, pp. 1306-1311, November, 1975.

[7] N. Abramson,'The Throughput of Packet Broadcasting Channels", IEEE Transactions on Communications, vol. COM-25, No. 1, January 1977.

[8] G. L. Choudhury, S. S. Rappaport, "Diversity ALOHA - A Random Access Scheme for Satellite Communications", IEEE Transactions on Communications, vol. COM-31, No. 3, March 1983.

[9] E. Casini,R. De. Gaudenzi, O. d. Rio Herrero, "Contention Resolution Diversity Slotted ALOHA (CRDSA): An Enhanced Random Access Scheme for Satellite Access Packet Networks", IEEE Transactions on Wireless Communications, vol. 6, no. 4, pp. 1408-1419, April 2007.

[10] O. d. Rio Herrero, R. de Gaudenzi,"A High-Performance MAC Protocol For Consumer Broadband Satellite Systems", Proceedings of the 27th International Communications Satellite Systems Conference (ICSSC), June 1st-4th, 2009, Edinburgh, Scotland.

[11] O. d. Rio Herrero, R. De Gaudenzi, "A high efficieincy scheme for quasireal-time satellite mobile messaging systems", Proceedings of the 10th International Workshop on Signal Processing for Space Communications, SPSC 2008, pp. 1-9, Oct 6th-8th, 2008, Rhodes Island, Greece.

[12] R. De Gaudenzi, O. d. Rio Herrero, "Advances in Random Access Protocols for Satellite Networks", Proceedings of the International Workshop on Satellite and Space Communications (IWSSC), Sept. 10th-11th, 2009, Siena, Italy.

[13] G. Liva, "A Slotted ALOHA Scheme Based on Bipartite Graph Optimization", 8th international ITG conference on source and channel coding SCC, Jan 18th - 21st, 2010, Siegen, Germany.

[14] G. Liva, "Graph-based Analysis and Optimization of Contention Resolution Diversity Slotted ALOHA", accepted for publication in IEEE transactions on communication, 2010. 